EPJ Web of Conferences 57, 01003 (2013)

DOI: $10.1051 /$ epjconf/20135701003

(C) Owned by the authors, published by EDP Sciences, 2013

\title{
Precision studies of Fermi gases using Bragg spectroscopy
}

\author{
S. Hoinka ${ }^{1}$, M.G. Lingham ${ }^{1}$, K. Fenech ${ }^{1}$, H. Hu${ }^{1}$, M. Delehaye ${ }^{1,2}$, \\ P. Hannaford ${ }^{1}$ and C.J. Vale ${ }^{1, a}$ \\ ${ }^{1}$ Centre for Atom Optics and Ultrafast Spectroscopy, Swinburne University of Technology, \\ Melbourne 3122, Australia \\ ${ }^{2}$ Departement de Physique, École Normale Supérieure, 24 rue Lhomond, 75005 Paris, \\ France
}

\begin{abstract}
We have used Bragg spectroscopy to make precise measurements of a number of properties of a strongly interacting Fermi gas. These include the dynamic spin and density response functions, the static structure factors and Tan's universal contact parameter. We also present a technique for obtaining the homogeneous response functions and contact using measurements on a trapped (inhomogeneous) system.
\end{abstract}

\section{INTRODUCTION}

Ultracold Fermi gases near Feshbach resonances have opened the way for new explorations of universal physics in strongly coupled fermion systems [1]. These systems are characterised by low temperatures and unitarity limited elastic collisions where $s$-wave scattering dominates and the scattering length $a$ exceeds all relevant length scales of the system $[2,3]$. Consequently $a$ plays no role in the physics and the system properties depend only on the density and temperature. When combined with our ability to precisely perturb and image cold atoms, these systems become ideal platforms to obtain data on physical parameters that are difficult to calculate for strongly coupled systems.

This paper describes recent measurements on the properties of ultracold Fermi gases near a Feshbach resonance. We use Bragg spectroscopy to make precise measurements of the dynamic density-density and spin response functions from which we determine the dynamic and static structure factors as well as Tan's universal contact parameter [4]. These parameters characterise the dynamic and static system properties and are significant for strongly interacting Fermi gases where exact theoretical calculations are presently unavailable. Our experiments provide new benchmarks for the contact parameter with error bars small enough to distinguish between several recently established theoretical predictions.

\section{BRAGG SPECTROSCOPY}

The starting point for our experiments is an evaporatively cooled cloud of ${ }^{6} \mathrm{Li}$ atoms, held in an optical dipole trap and prepared in an equal mixture of the $\left|F=1 / 2, m_{F}= \pm 1 / 2\right\rangle$ spin states (labelled $|\uparrow\rangle$ and $|\uparrow\rangle$ ) [5]. Clouds are cooled at a magnetic field of $833 \mathrm{G}$ corresponding to the pole of a broad Feshbach resonance between these spin states. Typical clouds contain $N_{\sigma} \sim 3 \times 10^{5}$ atoms per spin state at a temperature of $T / T_{F} \approx 0.10$ where the trapped Fermi energy is given by $E_{F}=k_{B} T_{F}=\left(6 N_{\sigma}\right)^{1 / 3} \hbar \bar{\omega}, k_{B}$ is Boltzmann's constant and $\bar{\omega}$ is the geometric mean harmonic confinement frequency. Bragg scattering

ae-mail: cvale@swin.edu.au

This is an Open Access article distributed under the terms of the Creative Commons Attribution License 2.0, which permits unrestricted use, distribution, and reproduction in any medium, provided the original work is properly cited. 


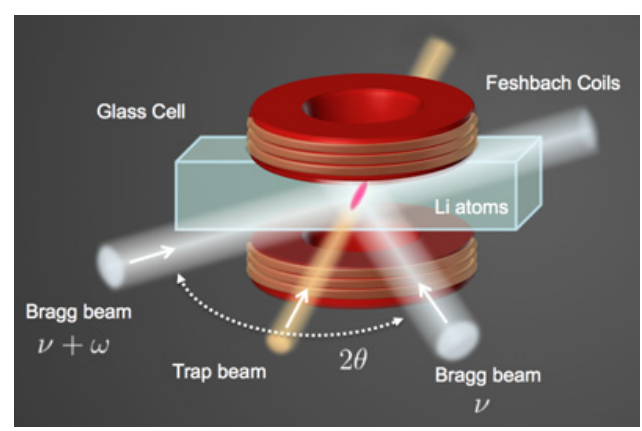

Figure 1. Experimental setup for Bragg scattering. A cloud of ${ }^{6} \mathrm{Li}$ atoms is cooled and trapped in a single beam optical dipole trap and then illuminated by two Bragg lasers intersecting at an angle of $2 \theta$.

is achieved by illuminating the atom cloud with two laser beams, intersecting at an angle of $2 \theta=84^{\circ}$ with a small tunable frequency difference $\omega$. A diagram of the basic experimental setup is shown in Fig. 1 below.

Bragg scattering involves the stimulated absorption-emission of a photon from one laser beam to the other beam, imparting momentum $\hbar k$, where $k=(4 \pi / \lambda) \sin \theta$, and energy $\hbar \omega$ to the scatterer. In a single particle picture, resonant Bragg scattering occurs when the energy difference between the two lasers $\hbar \omega$ is equal to the change in kinetic energy of the particle being scattered. This allows us to define the atomic recoil frequency $\omega_{r}=\hbar k^{2} /(2 m)$ where $m$ is the mass of a single atom and $\lambda=671 \mathrm{~nm}$ is the wavelength of the Bragg lasers. Note that the mass of the particles comes into the resonance condition which leads to the interesting possibility that molecules or pairs with twice the atomic mass, can be distinguished from atoms in the frequency dependence of the Bragg response.

Bragg spectroscopy is performed by illuminating trapped atom clouds with a short (typically $100 \mu \mathrm{s}$ ) pulse from the Bragg lasers and measuring the momentum imparted to the atoms as a function of the frequency difference $\omega$ between the two Bragg laser beams. To determine the momentum imparted we release the atom cloud from the trap immediately after the Bragg pulse, allow the atoms to expand freely for a short time of flight and then take an absorption image from which we determine the centre of mass displacement resulting from the Bragg pulse. This is directly proportional to the momentum transferred [6] which, in the limit of low intensity, is proportional to the imaginary part of the linear response function [7]. This is related to the dynamic structure factor $S(k, \omega)$ via the fluctuation dissipation theorem [8]. At high momentum and low temperature $k>k_{F}, \lambda_{d B}^{-1}$, where $\lambda_{d B}$ is the thermal de Broglie wavelength, the response becomes directly proportional to $S(k, \omega)$. Our measurements are all performed in this low Bragg intensity, high momentum regime meaning that the centre of mass displacements provide a direct measure of the dynamic structure factor.

\subsection{Density-density response}

Bragg spectroscopy with cold atoms has traditionally been used to measure density-density response functions and static structure factors in atomic Bose-Einstein condensates [9, 10] and Fermi gases [6]. In the case of two-component Fermi gases, the Bragg laser frequency can be set so that the coupling to atoms in each spin state is almost identical, resulting in a perturbation of the total density $\hat{\rho}(k)=\hat{\rho}_{\uparrow}(k)+\hat{\rho}_{\downarrow}(k)$. In this case one is sensitive to the imaginary part of the density-density response function $\chi_{\rho \rho}^{\prime \prime}(k, \omega)$. At high momentum transfer and low temperature this is equivalent to a measure of the dynamic structure factor $S(k, \omega)$.

Figure 2 shows a measurement of the dynamic density structure factor for a gas prepared at unitarity (the pole of the Feshbach resonance) at a temperature of $0.08 T_{F}$. Due to a number of technical 


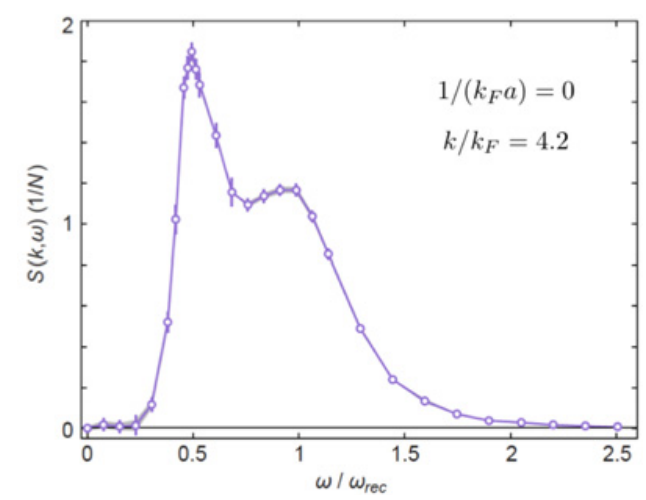

Figure 2. Bragg spectrum of a unitary Fermi gas at a temperature of $0.08 T_{F}$ and Bragg momentum of $k=4.2 k_{F}$.

and procedural improvements, we can now acquire Bragg spectra with far greater accuracy. This data has been normalised using the $f$-sum rule which gives $S(k, \omega)$ in units of $1 / N$. The spectrum shows a sharp feature at $\omega_{r} / 2$ corresponding to the scattering of pairs and a broader feature centred around $\omega_{r}$ arising from the scattering of single atoms. The pair excitation peak is very striking considering that, at unitarity, the two-body binding energy is zero and no 'true' molecules exist. We note that this peak at $\omega_{r} / 2$ emerges very rapidly when the temperature is decreased below the critical temperature for superfluidity $T_{c}\left(\simeq 0.2 T_{F}\right.$ in a trapped gas [11]) and may therefore provide a clear signature of pair condensation. Such a signature is difficult to see in the density distribution obtained by absorption images [11].

Integrating the normalised Bragg spectrum over $\omega$ provides a measure of the static structure factor which we find to be $S(k)=1 / N \int S(k, \omega) d \omega=1.182 \pm 0.004$ representing a vast improvement in accuracy over our previous measurements. At the high momentum of our measurement we can use this result to determine Tan's universal contact parameter, $\mathcal{I}$ in a trapped gas. The contact parameterises the amplitude of the momentum distribution at large $k$ and is proportional to the two-body correlation function, $g_{\uparrow \downarrow}^{(2)}(r)$ for small $r$. Fourier transforming Tan's relation for $g_{\uparrow \downarrow}^{(2)}(r)$ provides a universal relation for the static structure factor involving the dimensionless contact parameter $\mathcal{I} /\left(N k_{F}\right)$ given below

$$
\frac{\mathcal{I}}{N k_{F}}=\frac{4 k}{k_{F}}\left(\frac{S(k)-1}{1-4 /(\pi k a)}\right) .
$$

We obtain $\mathcal{I} /\left(N k_{F}\right)=3.06 \pm 0.08$ at unitarity where the error bar includes the uncertainty in $k_{F}$ that arises from uncertainty in determining the atom number. This provides a new experimental benchmark which can distinguish between different theoretical predictions that have been published in recent years and have varied by as much as $\sim 10 \%$. Our results are in reasonable agreement with the many-body $t$-matrix calculations of the Camerino [12] and Munich [13] groups but are somewhat below the Nozierre-Schmitt-Rink calculations of the Swinburne group [14]. We note that our measurements were performed at $0.08 T_{F}$ so should only be compared with these finite temperature calculations.

\subsection{Spin response}

When the Bragg lasers are tuned close to the atomic transition frequencies we can vary the relative coupling strength to atoms in the two different spin states. It then becomes possible to probe the spin response or magnetic susceptibility of the system. Of particular interest is the case when the Bragg lasers are tuned halfway between the atomic transition frequencies from each of the two spin states which probes purely the spin response function. A simple way to understand this is to realise that when the Bragg laser detuning is $+\Delta$ from one state and $-\Delta$ from the other state, the light shift potentials will have equal magnitude but opposite sign. This is analogous to applying a magnetic field so that 


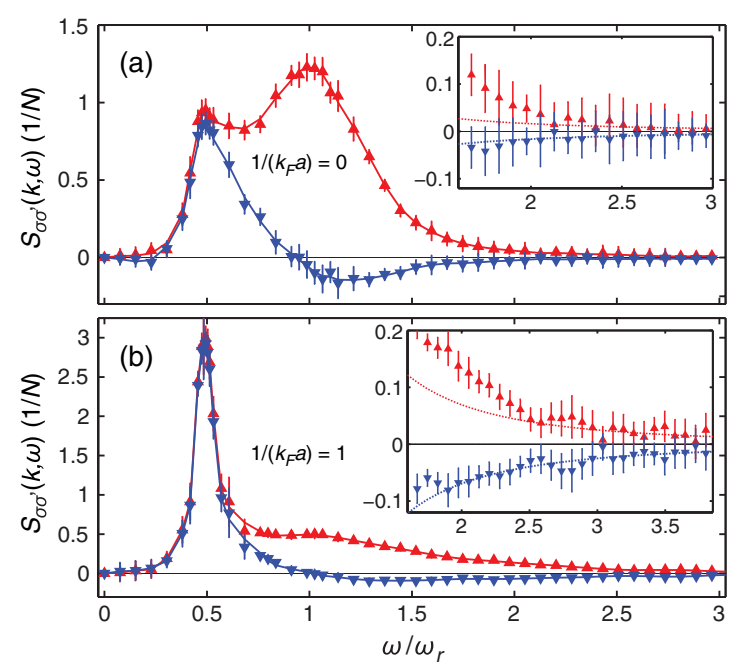

Figure 3. Spin-parallel and spin-antiparallel dynamic structure factors of a Fermi gas measured at (a) $1 /\left(k_{F} a\right)=0.0$, and, (b) at $1 /\left(k_{F} a\right)=1.0$. Red upright triangles are the spin-parallel structure factor $S_{\uparrow \uparrow}$ and blue inverted triangles are the spin anti-parallel response $S_{\uparrow \downarrow}$. Solid lines are a guide to the eye. Insets show zoomed in plots of the high frequency region where $S_{\uparrow \downarrow}(k, \omega)$ shows a universal tail proportional to $\omega^{-5 / 2}$. Thin lines are power law fits to $S_{\uparrow \downarrow}\left(k, \omega>2 \omega_{r}\right)$.

the perturbation couples to $\hat{S}_{z}(k)=\hat{\rho}_{\uparrow}(k)-\hat{\rho}_{\downarrow}(k)$. More generally, we can express the momentum transferred via the Bragg lasers, $\mathcal{P}(k, \omega)$, at arbitrary detunings in terms of the spin-parallel and spinantiparallel components of the dynamic structure factor, $S_{\uparrow \uparrow}(k, \omega)$ and $S_{\uparrow \downarrow}(k, \omega)$ respectively, as [15]

$$
\mathcal{P}(k, \omega) \propto\left(\frac{1}{\Delta_{\uparrow}^{2}}+\frac{1}{\Delta_{\downarrow}^{2}}\right) S_{\uparrow \uparrow}(k, \omega)+\frac{2}{\Delta_{\uparrow} \Delta_{\downarrow}} S_{\uparrow \downarrow}(k, \omega),
$$

where $\Delta_{\sigma}$ is the detuning from state $|\sigma\rangle$ and in a spin balanced gas the density (spin) structure factors are $S_{D+,(S-)}(k, \omega)=2\left[S_{\uparrow \uparrow}(k, \omega) \pm S_{\uparrow \downarrow}(k, \omega)\right]$. Combining measurements at different laser detunings it is straightforward to obtain the individual spin-parallel and spin-antiparallel response functions. These are shown for a gas at unitarity and on the BEC side of the Feshbach resonance in Fig. 3.

The features in $S_{\uparrow \uparrow}(k, \omega)$ and $S_{\uparrow \downarrow}(k, \omega)$ can now be better understood. At Bragg frequencies ranging from 0 to slightly above $\omega_{r} / 2$, both the spin-parallel and spin-antiparallel components are almost identical for both interaction strengths, $1 /\left(k_{F} a\right)=0$ and 1 . Within this frequency range the measured response is entirely dominated by the scattering of spin-up/spin-down pairs. Each pair contains a spin-up and spin-down particle, and, since the response is independent of the combination of spin states being probed, the density-density correlations for either combination of spin states are the same.

At higher Bragg frequencies the spin-parallel and spin-antiparallel response functions become very different with $S_{\uparrow \uparrow}(k, \omega)$ peaking near $\omega_{r}$ while $S_{\uparrow \downarrow}(k, \omega)$ becomes negative for $\omega \sim \omega_{r}$. The positive peak in $S_{\uparrow \uparrow}\left(k, \omega \approx \omega_{r}\right)$ is primarily due to the autocorrelation term arising from single particle scattering. This is not present in $S_{\uparrow \downarrow}(k, \omega)$ which always involves correlations between different particles. $S_{\uparrow \downarrow}(k, \omega)$ becomes negative at high frequencies which reveals an interesting feature of the relative dynamics of spin-up/spin-down particles. A high frequency fluctuation with momentum $k$ of the spin-up density leads to an out-of-phase fluctuation of the spin-down density. These anti-correlations suppress the high frequency response in the density channel but enhance the spin response.

For $\omega \gg \omega_{r}, S_{\uparrow \uparrow}(k, \omega)\left\{S_{\uparrow \downarrow}(k, \omega)\right\}$ is predicted to display a universal high-frequency tail proportional to $+\{-\} \mathcal{I} / \omega^{5 / 2}$, where $\mathcal{I}$ is Tan's contact parameter [16, 17]. The insets of Fig. 3 show 


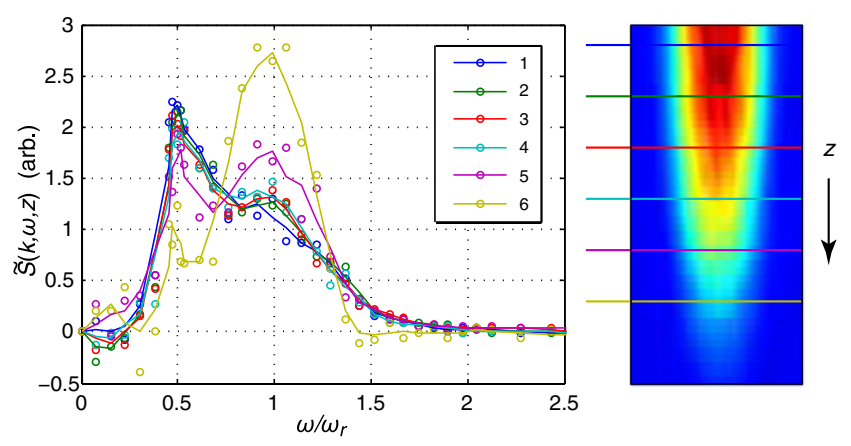

Figure 4. Bragg spectra, $\tilde{S}(k, \omega, z)$, showing the centre of mass displacement at different longitudinal positions in a unitary Fermi gas. Coloured spectra in the left image are made up by analysing 7 pixel wide slices centred around the coloured horizontal lines shown on the 2D-density profile on the right. Spectra near the cloud centre are dominated by a large pairing peak at $\omega_{r} / 2$ whereas spectra near the cloud edges are dominated by the atomic peak. Solid lines are a guide to the eye.

zoomed in plots of $S_{\uparrow \uparrow}(k, \omega)$ and $S_{\uparrow \downarrow}(k, \omega)$ in this high frequency range. Despite the relatively small signal, the amplitude of $S_{\uparrow \downarrow}(k, \omega)$ decays in a fashion that is well described by an $\omega^{-5 / 2}$ dependence. A free power-law fit to the data (blue line) for $\omega>2 \omega_{r}$ yields $S_{\uparrow \downarrow}(k, \omega) \propto \omega^{-2.5 \pm 0.3}$ on the BEC side and $S_{\uparrow \downarrow}(k, \omega) \propto \omega^{-2.7 \pm 0.8}$ at unitarity, consistent with the universal $\omega^{-5 / 2}$ dependence.

\section{HOMOGENEOUS RESPONSE}

In the final section of this paper we describe a method to obtain the homogeneous response function using spatially resolved measurements on a trapped (inhomogeneous) atom cloud. This idea extends the methods described by Ho and Zhou [18] and experimentally implemented by the Salomon group at École Normale Supérieure [19]. The basic idea is to apply a uniform Bragg pulse perpendicular to the long $(z)$ axis of an elongated cloud and spatially resolve the response in different regions. This gives a result integrated over two spatial dimensions rather than three as for bulk measurements.

In Fig. 4 we show Bragg spectra obtained at different $z$ positions through the cloud. These spectra, labelled $\tilde{S}(k, \omega, z)$, are proportional to the dynamic structure factor integrated over $x$ and $y$. While the cloud is in thermal equilibrium prior to the Bragg pulse each spectrum is obtained in a region of different peak density. Moving away from the cloud centre effectively lowers the local Fermi energy $\left(\epsilon_{F} \propto n^{2 / 3}\right)$ such that the relative temperature $\left(T / T_{F}\right)$ is higher than at the cloud centre.

Near the cloud centre the spectra are dominated by a large (pairing) peak at $\omega_{r} / 2$ whereas in the cloud wings the pairing feature diminishes and the broader peak centred around $\omega_{r}$ dominates the response. These spatially resolved spectra provide additional information over bulk measurements and can be used to obtain the local value of $S(k, \omega)$ along the axis of the trap as follows. Assuming $S(k, \omega)$ satisfies the local density approximation (LDA), which we expect as all measurements are performed at high momentum and are therefore sensitive to short-range spatial correlations, measurements of $\tilde{S}(k, \omega, z)$ can be expressed as a density weighted measurement of the homogeneous structure factor integrated over the density profile in the $x$ and $y$ directions, i.e.

$$
\tilde{S}(k, \omega, z)=\frac{1}{\tilde{n}(z)} \int S(k, \omega, \mu[x, y, z], T) n(\mu[x, y, z], T) d x d y
$$

where $\mu[x, y, z]=\mu_{0}-V(x, y, z)$ is the local chemical potential, $V(x, y, z)$ is the confining potential and $T$ is the cloud temperature. As shown in ref [18] for a purely harmonic trapping potential, the integral over $d x d y$ can be rewritten as an integral over the chemical potential $d \mu$. Making this substitution in Eq. (3) and differentiating both sides with respect to $z$ one finds an expression which 


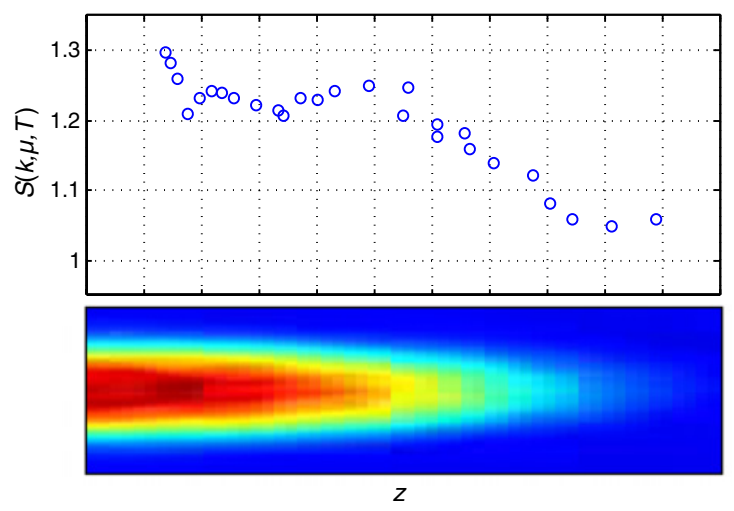

Figure 5. Homogeneous static structure factor along the axis of a unitary Fermi gas as described in the text.

relates the local structure factor $S(k, \omega, \mu[0,0, z], T)$ to the derivative of $\tilde{S}(k, \omega, z)$. When combined with the Gibbs-Duhem equation we obtain the following simple expression for the homogeneous structure factor along the axis of the trap

$$
S(k, \omega, \mu[0,0, z], T)=\frac{\partial(\tilde{S}[k, \omega, \tilde{n}(z)] \tilde{n}(z))}{\partial \tilde{n}(z)}
$$

where we have rewritten $\tilde{S}(k, \omega, z) \equiv \tilde{S}[k, \omega, \tilde{n}(z)]$ as a function of the local (integrated) density $\tilde{n}(z)$ as this is the origin of the $z$ dependence. This simple relation connects the value of the homogeneous structure factor along the axis of the trap to the derivative of the integrated structure factor and density. We note that this procedure is completely general and can be used to obtain the homogeneous value of any parameter which satisfies the local density approximation.

We can then obtain the homogeneous dynamic and static response functions as well as the contact. In Fig. 5 below we show the homogeneous static structure evaluated for a selection of slice thicknesses from 10 up to 24 pixels in width (the total cloud half width is approximately 55 pixels). Relatively thick slices were chosen to minimise noise as this process involves taking a derivative. Despite this, a clear trend is visible with the static structure factor starting off $\sim 1.25$ near the cloud centre and dropping down close to 1 at the cloud edges. Interactions increase the interparticle correlations most in the high density central part of the cloud where the relative temperature is lowest.

This procedure becomes susceptible to noise for thinner slices particularly near the wings and also the centre of the cloud. In the wings there are relatively few atoms so the signal to noise ratio decreases; however, in the centre of the cloud the density changes are very small and, since $\partial \tilde{n}(z)$ appears in the denominator of Eq. (4), this has a large effect on the signal. This data could also provide the temperature dependence of the homogeneous contact parameter; however at this stage the data is still too noisy to make a meaningful measurement with high spatial resolution.

\section{CONCLUSIONS}

In summary we have shown that Bragg spectroscopy is a useful tool for making precise measurements of the properties of strongly interacting Fermi gases. We have used it to make the most precise measurements to date of the contact parameter in a trapped Fermi gas and have presented a method for obtaining the spin response functions as well as the density-density response. Finally we have shown how spatially resolved measurements on a trapped cloud can be used to make a measure of the homogeneous values of the static structure factor or other variables that satisfy the LDA. 
All measurements reported in this work used a Bragg momentum that was large compared to the Fermi momentum. In this regime we probe single particle excitations which we interpret as the scattering of pairs of free atoms. This makes our measurements sensitive to universal short-range quantities such as the universal contact parameter. In future we will extend our studies into the low momentum regime to probe the collective response that should enable precise measurements of the pairing gap [20], speed of sound [8] and the frequency dependent shear viscosity [21].

\section{References}

[1] S. Giorgini, L. P. Pitaevskii, and S. Stringari, Rev. Mod. Phys. 80, 1215 (2008).

[2] H. Heiselberg, Phys. Rev. A 63, 043606 (2001).

[3] T.-L. Ho, Phys. Rev. Lett. 92, 090402 (2004).

[4] S. Tan, Ann. Phys. (N.Y.) 323, 2952, ibid. 2971, ibid. 2987 (2008).

[5] J. Fuchs et al., J. Phys. B: At. Mol. Opt. Phys. 40, 4109 (2007).

[6] G. Veeravalli, E. Kuhnle, P. Dyke, and C. J. Vale, Phys. Rev. Lett. 101, 250403 (2008).

[7] A. Brunello et al., Phys. Rev. A 64, 063614 (2001).

[8] L. Pitaevskii, and S. Stringari, Bose-Einstein condensation, Clarendon Press, Oxford (2003).

[9] D. M. Stamper-Kurn et al., Phys. Rev. Lett. 83, 2876 (1999).

[10] J. Steinhauer et al., Phys. Rev. Lett. 88, 120407 (2002).

[11] M. J. H. Ku, A. T. Sommer, L. W. Cheuk, and M. W. Zwierlein, Science 335, 563 (2012).

[12] F. Palestini, A. Perali, P. Pieri, and G. C. Strinati, Phys. Rev. A 82, 021605 (2010).

[13] T. Enss, R. Haussmann, and W. Zwerger, Ann. Phys. 326, 770 (2011).

[14] H. Hu, X.-J. Liu, and P. D. Drummond, New J. Phys. 13, 035007 (2011).

[15] S. Hoinka, M. Lingham, M. Delehaye, and C. J. Vale, Phys. Rev. Lett. 109, 050403 (2012).

[16] H. Hu, and X.-J. Liu, Phys. Rev. A 85, 023612 (2012).

[17] Y. Nishida, Phys. Rev. A 85, 053643 (2012).

[18] T.-L. Ho and Q. Zhou, Nat. Phys. 6, 131 (2010).

[19] S. Nascimbene, N. Navon, K. J. Jiang, F. Chevy, and C. Salomon, Nature 463, 1057 (2010).

[20] A. Minguzzi, G. Ferrari, Y. Castin, Eur. Phys. J. D 17, 49 (2001).

[21] E. Taylor and M. Randeria, Phys. Rev. A 81, 053610 (2010). 